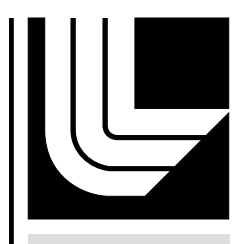

LAWRENCE LIVERMORE NATIONAL LABORATORY

TARANTULA 2011 in JWL++

P. C. Souers, D. Haylett, P. Vitello

October 27, 2011 
This document was prepared as an account of work sponsored by an agency of the United States government. Neither the United States government nor Lawrence Livermore National Security, LLC, nor any of their employees makes any warranty, expressed or implied, or assumes any legal liability or responsibility for the accuracy, completeness, or usefulness of any information, apparatus, product, or process disclosed, or represents that its use would not infringe privately owned rights. Reference herein to any specific commercial product, process, or service by trade name, trademark, manufacturer, or otherwise does not necessarily constitute or imply its endorsement, recommendation, or favoring by the United States government or Lawrence Livermore National Security, LLC. The views and opinions of authors expressed herein do not necessarily state or reflect those of the United States government or Lawrence Livermore National Security, LLC, and shall not be used for advertising or product endorsement purposes.

This work performed under the auspices of the U.S. Department of Energy by Lawrence Livermore National Laboratory under Contract DE-AC52-07NA27344. 


\title{
TARANTULA 2011 in JWL++
}

\author{
Lawrence Livermore National Laboratory, Livermore, CA 94550
}

LLNL-TR-509132

by P. Clark Souers, Dan Haylett and Peter Vitello

October 27, 2011

Using square zoning, the 2011 version of the kinetic package Tarantula matches cylinder data, cylinder dead zones, and cylinder failure with the same settings for the first time. The key is the use of maximum pressure rather than instantaneous pressure. Runs are at 40, 200 and $360 \mathrm{z} / \mathrm{cm}$ using $\mathrm{JWL}++$ as the host model. The model also does run-to-detonation, thinpulse initiation with a P-t curve and air gap crossing, all in cylindrical geometry. Two sizes of MSAD/LX-10/LX-17 snowballs work somewhat with these settings, but are too weak, so that divergent detonation is a challenge for the future. Butterfly meshes are considered but do not appear to solve the issue.

\section{Summary Results with the Same Settings}

Tarantula is a kinetic package designed for reactive flow codes, which seeks to model detonation, failure and corner-turning in cylindrical geometry. In this report, we use JWL++ as the base model, but it can run inside any Reactive Flow model. Four tests must be passed with the same settings. For ambient LX-17, we require:

\section{$6.25 \mathrm{~mm}$-radius copper cylinder detonation velocity}

$12.5 \mathrm{~mm}$-radius copper cylinder detonation velocity small-radius (eg $2.5 \mathrm{~mm}$ ) copper cylinder failure give the right edge distance to breakout in the double cylinder (see Figure 1a).

The details of the four tests are listed in Table 1a.

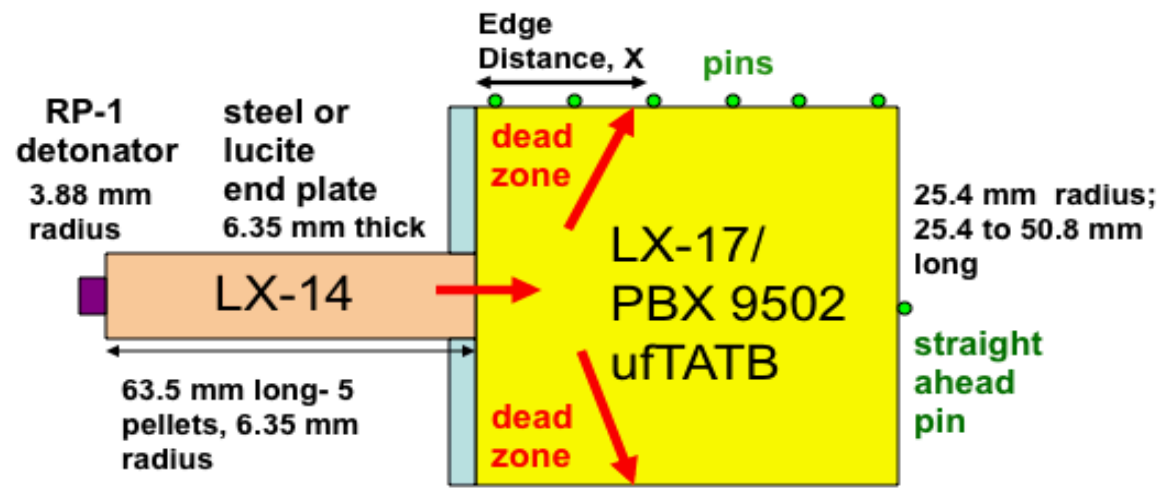

Figure 1a. Double cylinder corner-turning test, where we measure the breakout distance $x$ caused by dead zone formation. 
Table 1a. Cylindrical tests for calibrating Tarantula.

\begin{tabular}{|c|c|c|c|}
\hline Nom. density (g/cc) & $\mathrm{LX}-17$ & PBX 9502 & ufTATB \\
\hline Ambient & 1.900 & 1.890 & 1.800 \\
\hline Cold & 1.920 & 1.907 & 1.822 \\
\hline Hot & 1.874 & 1.867 & 1.778 \\
\hline \multicolumn{4}{|c|}{ Detonation Velocity $(\mathrm{mm} / \mu \mathrm{s})$} \\
\hline & $\mathrm{LX}-17$ & PBX 9502 virgin & ufTATB \\
\hline Small cylinders & 7.475-7.495 & $7.475-7.495$ & 7.44-7.46 \\
\hline Large Cylinders & 7.555-7.575 & 7.555-7.575 & 7.48-7.51 \\
\hline \multicolumn{4}{|c|}{ Small Cylinders: $6.25 \mathrm{~mm}$ radius, $2.0 \mathrm{~mm}$ wall } \\
\hline \multicolumn{4}{|c|}{ Large Cylinders: $12.5 \mathrm{~mm}$ radius, $2.5 \mathrm{~mm}$ wall } \\
\hline \multicolumn{4}{|c|}{ Failure Radius [Wall Thickness] $(\mathrm{mm})$} \\
\hline & $\mathrm{LX}-17$ & PBX 9502 & UTTATB \\
\hline & copper cylinder & copper cylinder & bare ratestick \\
\hline Ambient & $2.5[2.0]$ & $2.5[1.0]$ & \\
\hline Cold & $4.5[1.5]$ & $4.5[1.0]$ & 1.0 fail; 3.0 go \\
\hline Hot & $1.0[2.0]$ & $1.0[1.0]$ & \\
\hline \multicolumn{4}{|c|}{ Break-Out Distance Corner-Turn (mm) } \\
\hline & $\mathrm{LX}-17$ & PBX 9502 & ufTATB \\
\hline Ambient & 13-19 & $9-13$ & $\sim 0$ \\
\hline Cold & $40-45$ & $22-31$ & $\sim 0$ \\
\hline Hot & $7-13$ & $7-9$ & 0 \\
\hline Threshold $\mathrm{P}_{\mathrm{o}}(\mathrm{Mb})$ & $L X-17$ & PBX 9502 & ufTATB \\
\hline Ambient & 0.075 & 0.065 & 0.037 \\
\hline Cold & 0.095 & 0.080 & 0.042 \\
\hline Hot & 0.055 & 0.050 & 0.030 \\
\hline
\end{tabular}

For several years, Tarantula did three of the four tests, but getting cylinder failure and dead zone formation with the same settings did not work. This year, we changed pressure $(P+Q)$ to maximum-achieved pressure in the rate and we are able to fit all four tests. A new history variable, Pmax_his, tracks the pressure $(P+Q)$ as it rises, then holds it constant at the maximum value when the pressure starts to decline. Zoning was done at 40, 200 and 360 zones $/ \mathrm{cm}$. There is a large difference in settings between 40 and $200 \mathrm{zones} / \mathrm{cm}$, but once even one setting is worked out, all others can be derived by small changes. The ambient LX-17 worked at 360 zones/cm with no change from 200 . The settings are listed in Table $1 \mathrm{~b}$.

The one thing that did NOT work well was the size of the dead zone in cold LX-17, which has the largest dead zones of the TATB group. The real dead zone is huge, about $40-50 \mathrm{~mm}$ down the side of the double cylinder. We routinely got $25 \mathrm{~mm}$ from the model and sometimes 
$35 \mathrm{~mm}$. We were never able to get the full large size. A typical $23 \mathrm{~mm}$ run is shown in Figure $1 b$, and the dead is not large enough.

Table 1b. Settings used for successful Tarantula tests. Monotonic $Q$ was used.

\begin{tabular}{|c|c|c|c|c|c|c|c|c|c|c|c|}
\hline & & zones/ & \multicolumn{3}{|c|}{ Pressure in $\mathrm{Mb}$} & & & \multicolumn{4}{|c|}{ Mb units for G's } \\
\hline explosive & Temperature & $\mathrm{cm}$ & Po & $\mathrm{P} 1$ & $\mathrm{P} 2$ & b1 & $\mathrm{b} 2$ & b3 & G1 & G2 & G3 \\
\hline LX-17 & cold & 40 & 0.095 & 0.23 & 0.35 & 3 & 2.7 & 0 & 1500 & 700 & 90 \\
\hline LX-17 & ambient & 40 & 0.075 & 0.18 & 0.32 & 3 & 2.7 & 0 & 1600 & 500 & 85 \\
\hline LX-17 & ambient & 200 & 0.075 & 0.25 & 0.35 & 2.7 & 1.0 & 0 & 700 & 36 & 85 \\
\hline LX-17 & ambient & 360 & 0.075 & 0.25 & 0.35 & 2.7 & 1.0 & 0 & 700 & 36 & 85 \\
\hline LX-17 & hot & 40 & 0.055 & 0.18 & 0.32 & 3 & 2.7 & 0 & 1600 & 650 & 85 \\
\hline PBX 9502 & ambient & 40 & 0.065 & 0.19 & 0.33 & 3 & 2.7 & 0 & 1600 & 500 & 88 \\
\hline PBX 9502 & ambient & 200 & 0.065 & 0.26 & 0.36 & 2.7 & 1.0 & 0 & 600 & 40 & 85 \\
\hline ufTATB & ambient & 40 & 0.037 & 0.14 & 0.28 & 3 & 2.7 & 0 & 1600 & 1850 & 155 \\
\hline ufTATB & ambient & 200 & 0.037 & 0.15 & 0.30 & 2.7 & 1.0 & 0 & 700 & 105 & 250 \\
\hline $\mathrm{Po}=\mathrm{P} ! \mathrm{off}=$ & $1=c 2=$ & $=1.5$ & & & & & & & & & \\
\hline
\end{tabular}

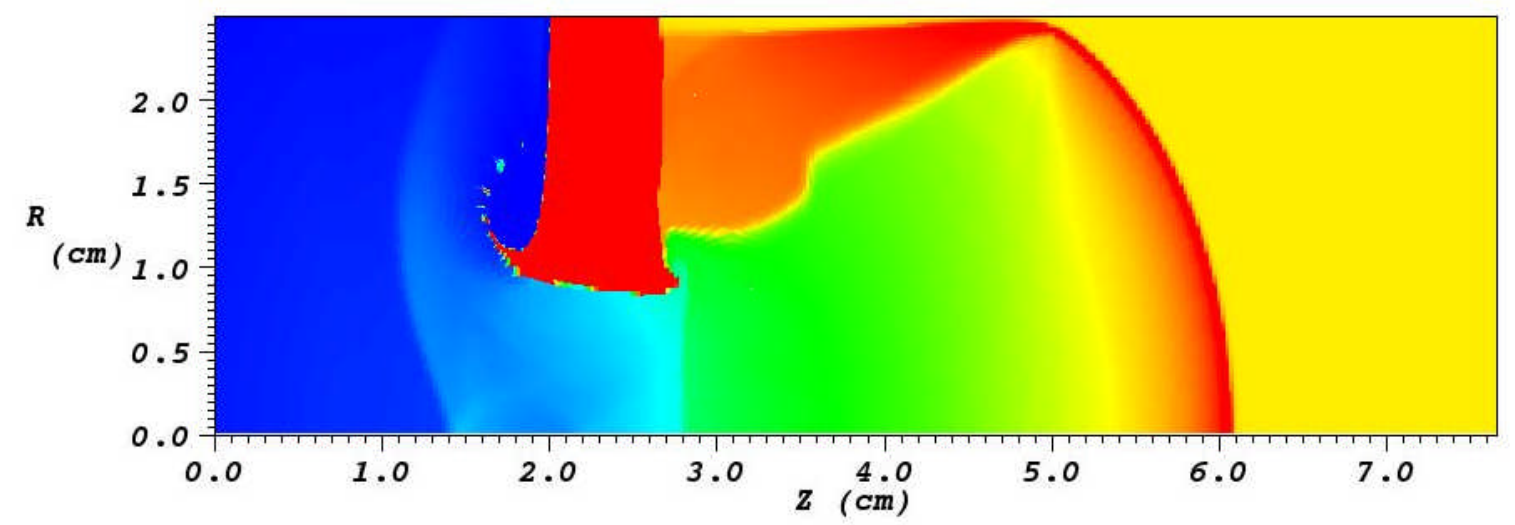

Figure $1 \mathrm{~b}$. Typical cold dead zone in LX-17. The edge distance of $23 \mathrm{~mm}$ is nowhere as large as the measured $40-50 \mathrm{~mm}$.

\section{The New 2011 Version of Tarantula}

In this version, pressure is changed to maximum pressure. The psychological barrier to be crossed for this next step was to add a history variable to the code. We define the variable $P_{\text {max_his }}$ as being the larger of two quantities

$$
P_{\text {max_his }}=\max \left(P_{\text {max_his }}, P+Q\right) \text {. }
$$


$P_{\text {max_his }}$ is only updated at the shock front where the monotonic artificial viscosity $Q>0.1^{*} P$, the actual pressure. If it detonates in a particular cell, we follow it from zero up to the top of the spike, where it stays. If it doesn't detonate, it still stays at the largest pressure value that was obtained.

The old rates are the same in the three regions listed below, where $P$ means $P+Q$.

$$
\begin{aligned}
\frac{d F}{d t} & =G_{1}\left[P_{\text {pmax_his }}-P_{\text {off } 1}\right]^{b_{1}}(1-F)^{c_{1}}, P_{0}<P<P_{1} \text {, initiation } \\
\frac{d F}{d t} & =G_{2}\left[P_{\text {pmax_his }}-P_{\text {off2 }}\right]^{b_{2}}(1-F)^{c_{2}}, P_{1}<P<P_{2} \text {, ramp - up / failure } \\
\frac{d F}{d t} & =G_{3}\left[P_{\text {pmax_his }}-P_{\text {off3 }}\right]^{b_{3}}(1-F)^{c_{3}}, P_{2}<P \text {, detonation }
\end{aligned}
$$

The above are separated with the same $P+Q$ pressure boundaries.

$$
\begin{aligned}
& P_{0} \text { below - no reaction; above initiation } \\
& P_{1} \text { below-initation; above ramp-up/failure } \\
& P_{2} \text { below-ramp-up/failure; above detonation }
\end{aligned}
$$

There is also a de-sensitization rate, which transforms the un-reacted explosive to an inert species with the same EOS as the un-reacted explosive, which effectively takes explosive out of the problem. This rate is applied for pressures less than $\mathrm{P}_{\mathrm{d}}$. This reaction rate is of the form

$$
\frac{d F_{d}}{d t}=G_{d} P_{\text {pmax_his }}^{b_{d}}(1-F) .
$$

The only problem where desensitization appears to definitely occur is Jackrabbit, where a weak shock goes through the plate while the detonation tries to run around it.

Turn-on for the model overall occurs when $\left(\rho / \rho_{o}-1-a\right)>0$ or if $P>P_{0}$, where $a$ is an input parameter. Once burning starts it continues regardless of the density. 
As an aside, if (1-F) to-the-first-power is used in JWL++ for the detonation region, then $\mathrm{G}_{3}$ will be close to the numbers we get from the inverse of the slope of the size effect. We tried this but found that the slope of the calculated size effect curve was wrong. We, therefore, retreated to the old empirical practice of using $(1-\mathrm{F})^{1.5}$ in the detonation region, which fits the data but has a larger $\mathrm{G}_{3}$.

The result of the Tarantula rate structure is to create two thresholds, as shown in Figure 2a. The first is starting at $P_{0}$ with detonation only for a pulse with more pressure than this. The second is the big jump in rate that leads to detonation going up or failure going down. The old quadratic rate used in JWL++, Ignition \& Growth and Linked CHEETAH is shown for comparison. This rate can do the size effect in detonation, failure or dead zones but each with $\underline{\text { a different setting. }}$.

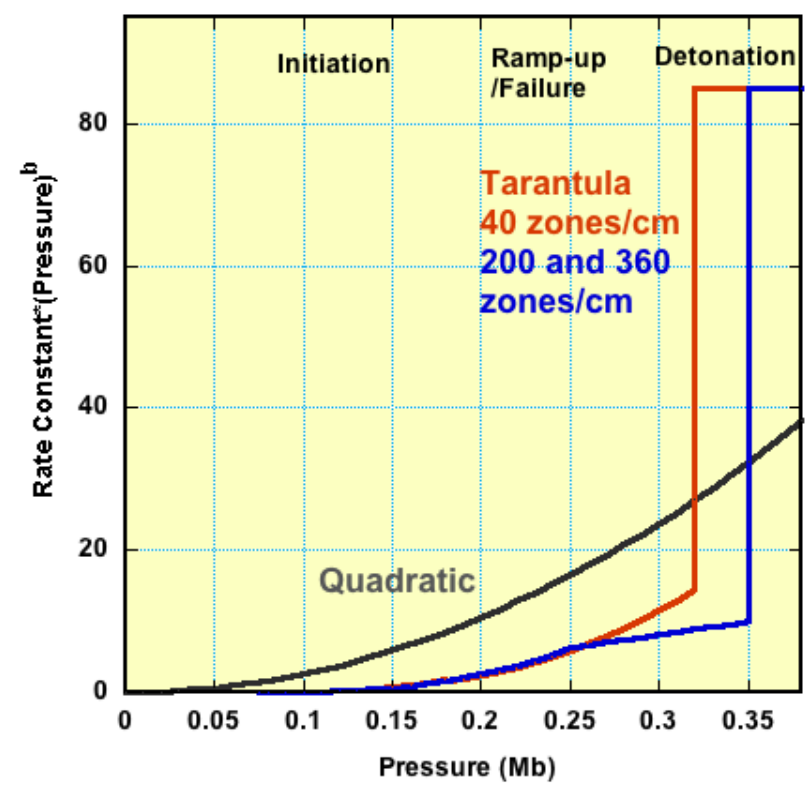

Figure 2a. Rates used in Tarantula as compared with the old quadratic curve.

\section{Other Observations in Running Tarantula}

1. This is the best feature of the new model: the mechanisms are now separated into pairs. Failure and dead zones appear to be both determined by the ramp-up/failure region as determined by $\mathrm{G}_{2}$. The $6.5 \mathrm{~mm}$ and $12.5 \mathrm{~mm}$ detonation velocities are both determined by $\mathrm{G}_{3}$ in the detonation region. This great result means that the 1-inch cylinder will not move even as we fiddle below $\mathrm{P}_{2}$. This feature allows simultaneous fitting of the basic four experiments. 
2. The hard part is getting the smallest cylinder to fail, which can always be achieved by increasing $P_{2}$. However, if $P_{2}$ gets too high, then the $6.5 \mathrm{~mm}$ cylinder will also fail.

3. The final settings are not unique but they lie as "islands", and there is little slack in the system to play with. The islands are fairly large at ambient and hot but small when cold, because the failure radius is larger. Cold is the most difficult to fit. Once on an island, the model responded not to the rate constant $\mathrm{G}$, but more to the integral of the rate constant with pressure.

4. Dead zones vary in shape from bananas to potatoes, with the latter being desirable. The bananas often have rounded tops, so that first breakout occurs too soon, even though the dead zone is large enough.

5. Going to qneg and Peter Vitello's $Q$ and tensor $Q$ did not improve the quality of the detonation fronts or the dead zones. It was not possible to get good-looking dead zones with tensor $Q$. Also, tensor $Q$ was extremely difficult to run with much worse zone tangling than any of the others, although a fix was found in every case.

\section{Obtaining Input Data}

The inverse radius equation for the size effect predicts an average detonation rate, $v$, given by

$$
v=\frac{-D^{2}}{\partial U_{s} / \partial\left(1 / R_{0}\right)}
$$

where $U_{S}$ is the detonation velocity for a explosive cylinder of radius $R_{0}$ and $D$ is the infiniteradius detonation velocity. If the size effect curve is straight, which LX-17 is to zeroth order, then the rate $v$ is constant, which is the justification for setting the Tarantula rate constant to be constant in the detonation region. $\mathrm{P}_{2}$, then, is the pressure at the failure point, which can be estimated for $L X-17$ using detonation velocity data.

$$
P_{2} \approx\left[\frac{U_{S}(\text { fail })}{D}\right]^{2}\left(1.2 P_{C j}\right)=\left(\frac{7.3}{7.66}\right)^{2}(1.2 * 0.28) \approx 0.31 \mathrm{Mb} \text { for } L X-17
$$


where $U_{s}($ fail) is the detonation velocity near failure for the smallest size cylinder, $D$ is the detonation velocity at infinite radius, $P_{\mathrm{cj}}$ is the CHEETAH C-J pressure and the "1.2" is an empirical way to add in the spike.

$P_{0}$ is the pressure threshold for initiation, which is measured from flyer impact studies. $P_{1}$ is in-between somewhere, with no definite mode of calculation. Presumably, it should be high enough that the run-to-detonation in initiation is too small to matter with the zoning being used. This is near $0.20 \mathrm{Mb}$ for LX-17 and ufTATB. POFF0 (see below for terms) is subtracted from pressure in the region 1 rate so as to make the rate zero at $P_{0}$. In theory, we want ramp-up to continuously flow from initiation at $\mathrm{P}_{1}$ and POFF1 can be used for that. We always set $\mathrm{G}_{3}$ constant for detonation, so POFF3 is zero. JWL++ does not mind discontinuities in the rate; it is similar to Ignition \& Growth in this regard.

The general modeling experience with LX-17 and PBX 9502 JWL's is summarized in the "Rule of Thumb": to zeroth order, the densities change with temperature but the detonation velocities, energy densities and rates stay the same. This occurs because the thermal lattice energy and the energy density change upon shrinkage or expansion almost exactly offset each other. This is close enough to have been of great help in laying out JWL models at different temperatures. The confined LX-17 data shown in Figure 4a is close to obeying the Rule of Thumb.

Figure 4b shows Campbell's famous recycled PBX 9502 bare ratesticks [Campbell] with EDC-35 data [Hutchinson] with the same composition. In box A, the Rule of Thumb works: we can lay down a single line. In box $B$, near failure, the points diverge and the Rule of Thumb does not work. This small-size divergence has not been seen in confined LX-17. Point $C$ is an ambient 4-inch diameter shot and it is slightly high, whereas point $D$ is way up. 


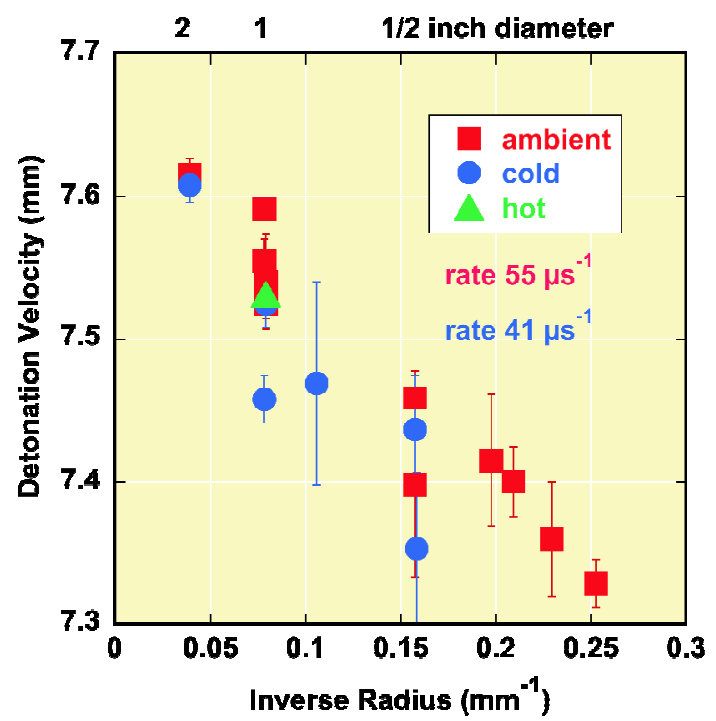

Table 4a. Seeking the Rule of Thumb: size effect curves for $L X-17$ cold and ambient with one hot point. The rates determined from the inverse slope are slightly different.

Figure $4 c$ shows the detonation velocities for ufTATB [Phillips, Souers1]. Copperconfined data shows a higher detonation rate than the bare ratesticks. The former data was measured with pins and the latter with a streak camera. We take the confined data as being the rate we want the model to use, but we also require failure for the $1 \mathrm{~mm}$-radius ratestick. The model, however, cannot describe the difference in slopes between the confined and unconfined explosive.

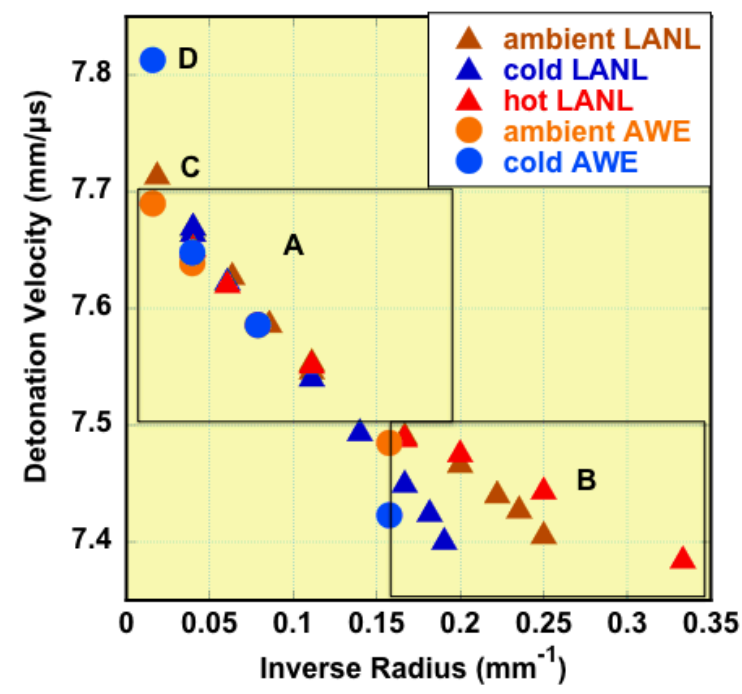

Figure 4b. Detonation velocity of PBX 9502 bare ratesticks at three temperatures with EDC 35 data cold and ambient. The Rule of Thumb is in trouble here. [Campbell]. 


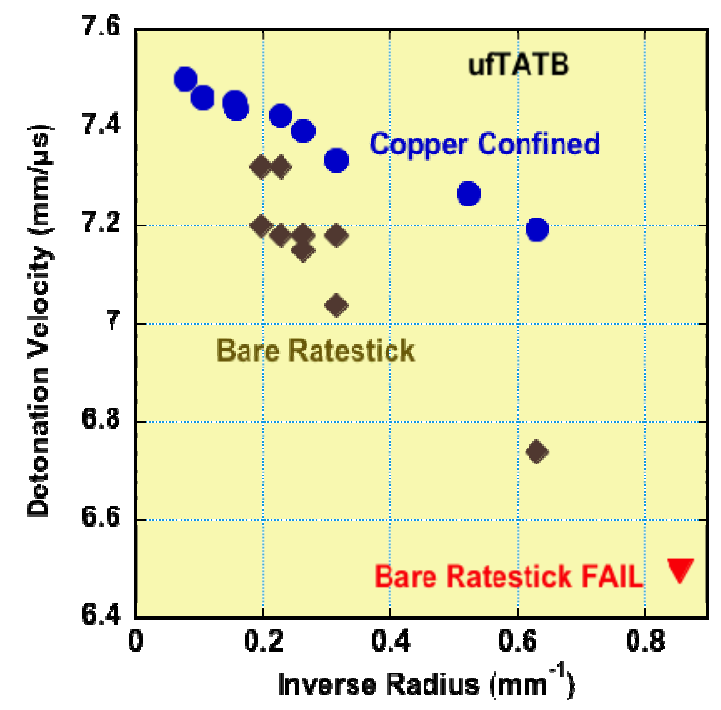

Figure 4c. The ufTATB size effect curves show different slopes for confined and unconfined. It is unlikely that any reactive flow model can handle this difference.

The initiation pressure threshold, $\mathrm{P}_{\mathrm{o}}$, has been measured by various means, with variable-velocity flyers being the most straight-forward. $\mathrm{P}_{\mathrm{o}}$ is the lowest pressure at which an explosive can run-to-detonation. It also assumes a very long sabot, which has the same cross-sectional area as the explosive. $P_{0}$ has not been measured as a function of temperature, so we take the densities instead $(1.920 \mathrm{~g} / \mathrm{cc}$ cold and $1.874 \mathrm{~g} / \mathrm{cc}$ hot $)$ and use Figure $4 d$ to estimate the threshold pressures. We use for $P_{\text {off2 }}$ the values $0.075,0.095$ and $0.055 \mathrm{Mb}$ ambient, cold and hot.

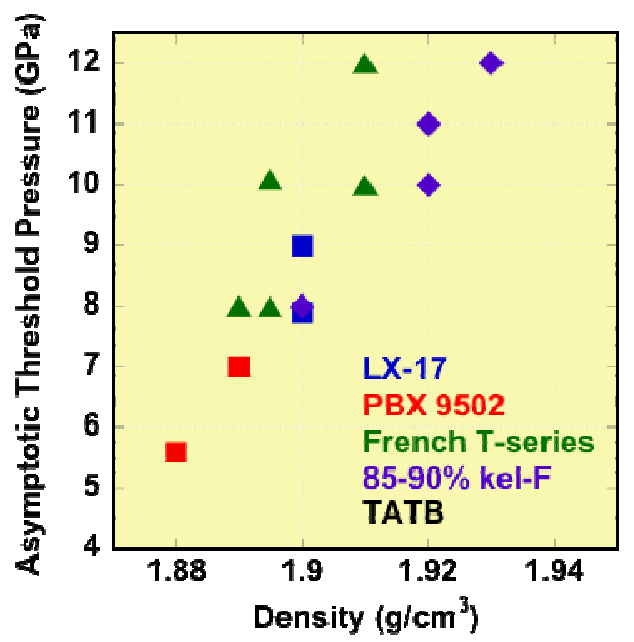

Figure 4d. Initiation threshold $\mathrm{P}_{\mathrm{o}}$ measured at room temperature for TATB explosives of various densities. We use density to simulate the effects of temperature. 
All boosters were done with simple JWL++. At 40 zones/cm, the zoning is always too coarse. The rate constant is set as high as possible; if set too high, the reactive flow strength actually decreases. At 360 zones/cm, the zoning is about right for HMX boosters, but too coarse for PETN. We have found, however, that even a bad JWL++ model is better than programmed burn, because it has a pressure spike and some detonation front curvature. In the hemisphere problem, the MSAD is explicitly modeled with extra zones in the X-direction at 40 zones/cm. At higher zoning, it is square in the MSAD. The MSAD is initiated in the LX16 and the aluminum flyer goes down the barrel to strike the booster.

"Fail" means that the edits with time will show a dying pressure, as shown in Figure 4e. The pseudocolor picture will change from blue to green to orange to yellow as the pressure falls.

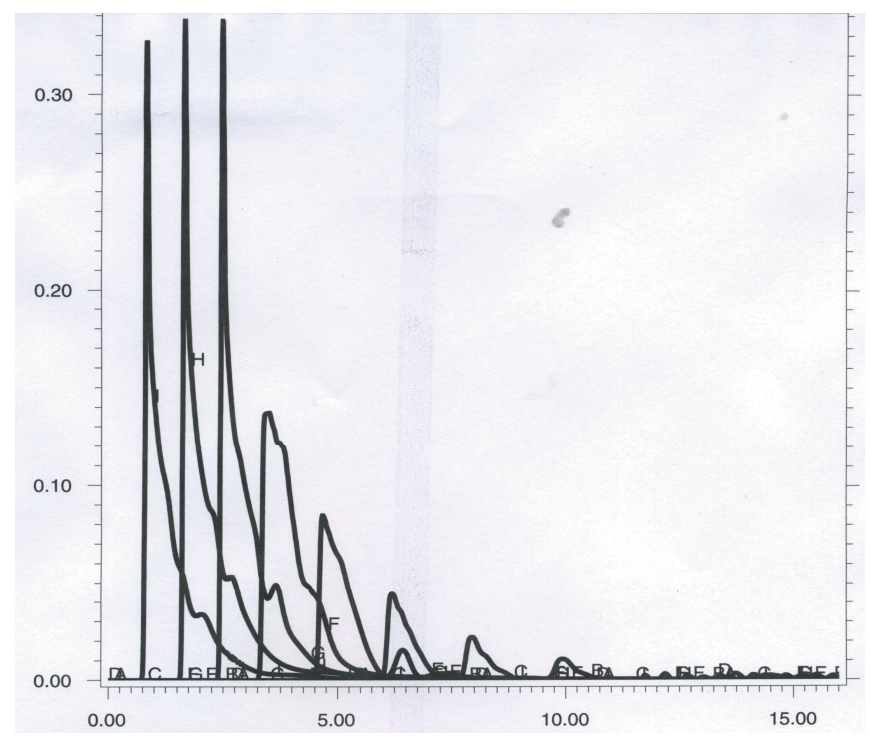

Figure 4e. Declining pressure spikes mean failure.

\section{Run-to-Detonation}

The initiation term, running from $P_{0}$ to $P_{1}$, is supposed to do run-to-detonation, even though we made no effort to use it this way. We now check to see how well it did. A typical set of pressure curves for ambient LX-17 at $11 \mathrm{GPa}$ and 200 zones/cm is shown in Figure $5 a$. 


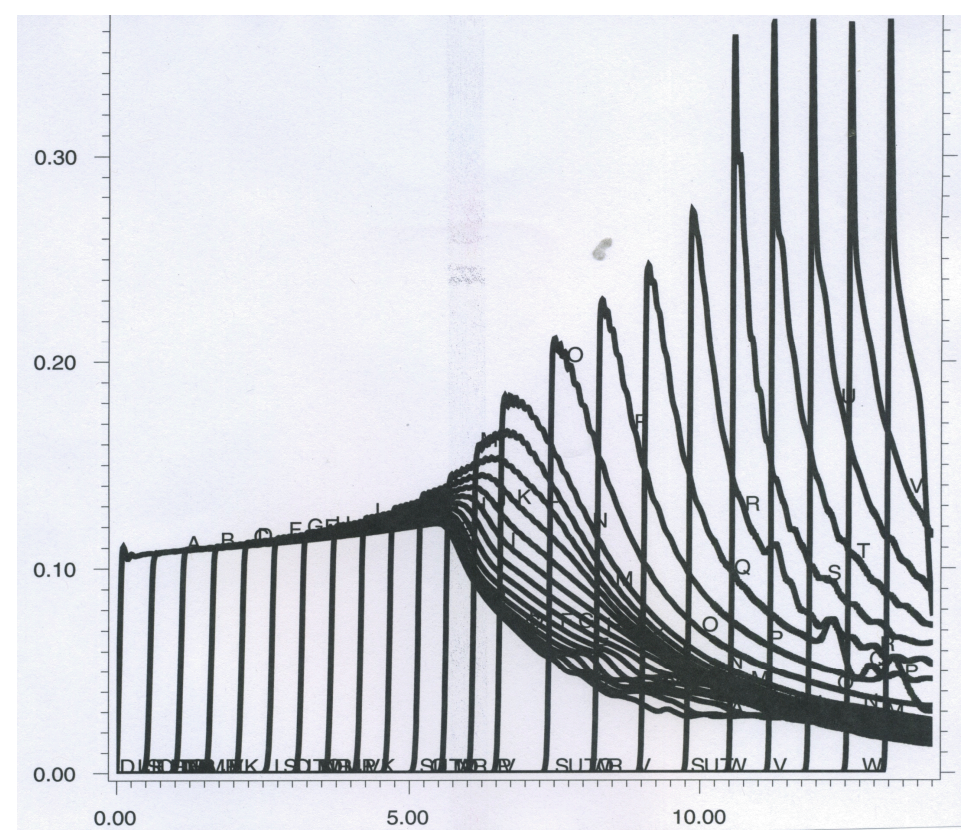

Figure 5a. Run-to-detonation curves for ambient LX-17 at $11 \mathrm{GPa}$ with a massive sabot.

We ask for $0.01 \mathrm{Mb}$ times for each edit point down the axis of the explosive and plot two results in Figure $5 \mathrm{~b}$ as a function of distance-versus-time. This $\mathrm{x}$-t plot shows two slopes and their intersection makes the time/distance for run-to-detonation. This is the same process used to get run distances and times from real data. The two slopes are present at 40 zones/cm although the difference is not great, and this difference increases with increased zoning.

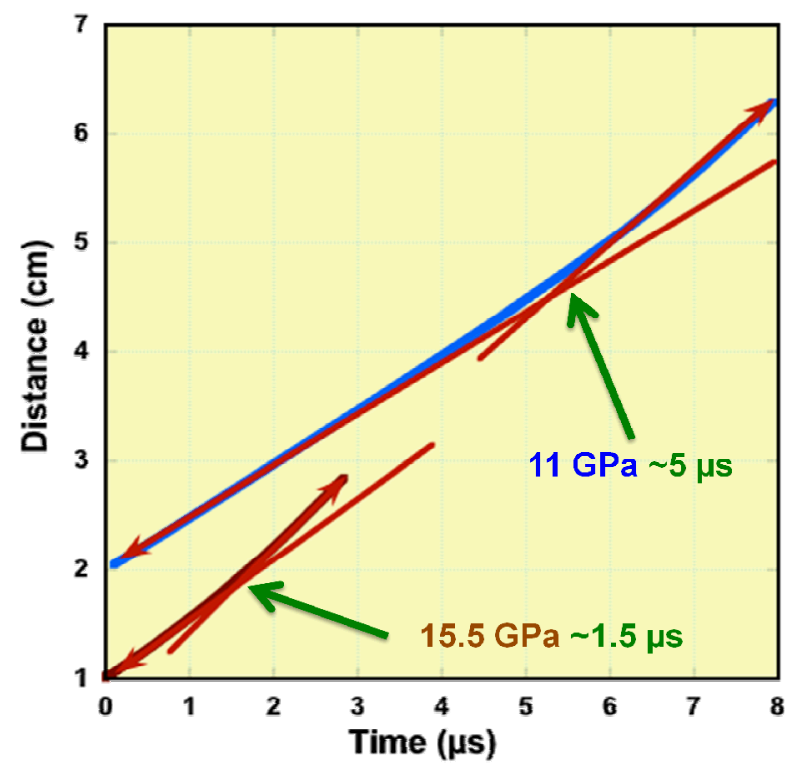

Figure $5 b$. Distance vs time plot for initiating explosive. 
Run-to-detonation times at three constant pressures are listed in Table 5a. The calculated values are close to the measured ones [Dallman, Gustavsen, Jackson]. It is important to note that the true delay is much smaller than is suggested by the data, because the initiating explosive is moving at a large fraction of the detonation velocity even at the start. The calculated real delays are about $0.02,0.05$ and $0.2 \mu$ s at $17.5,15.5$ and $11 \mathrm{GPa}$.

Table 5a. Run-to-detonation times ( $\mu$ s) for three constant pressures on the explosive.

\begin{tabular}{|c|c|c|c|}
\hline \multirow{2}{*}{ zones/ } & \multicolumn{3}{|c|}{ Pressure (GPa) } \\
\cline { 2 - 4 } $\mathrm{cm}$ & 17.5 & 15.5 & 11 \\
\hline 40 & $0.3-0.5$ & 0.9 & $5-6$ \\
\hline 200 & 0.5 & 1.5 & 5 \\
\hline 360 & 0.7 & 1.3 & \\
\hline measd & $\mathbf{0 . 6}$ & $\mathbf{0 . 9}$ & $\mathbf{4 . 5}$ \\
\hline
\end{tabular}

\section{50-50 Initiation}

Another initiation test is to see if the explosive follows the 50-50 P-tau curve, where $\mathrm{P}$ is pressure and $\tau$ is the pulse length applied by a flyer. As seen in Figure 6a, below the curve, the explosive does not detonate; if above the curve, it does [Honodel1, 2]. The bullet or thick sabot area lies far to the right on this plot, where $P_{0}$ is the minimum threshold pressure needed to start initiation. On the far left is the "thin-pulse" initiation region, where $\tau$ is small because the flyer is thin, so that the initiating pressure is high. At 200 zones $/ \mathrm{cm}$, the flyer is only one zone thick, so we cannot expect great modeling.

The data was taken with mylar/kapton flyers on LX-17, where both had radii of $12.7 \mathrm{~mm}$. We ran some these but most calculations were done with a $10 \mathrm{~mm}$ radius (for no good reason) and copper flyers. The reason for copper is that the calculations are better with the denser flyer as the flyers become thin. The thinnest flyer was $0.005 \mathrm{~cm}$, which is exactly one zone wide at 200 zones/cm. Even at 360 zones/cm, we are not close to having the desired 510 zones width in the flyer.

As seen in Figure 6a, the model does indeed do initiation at different pulse lengths, where only resolution limits the performance for thin flyers. 


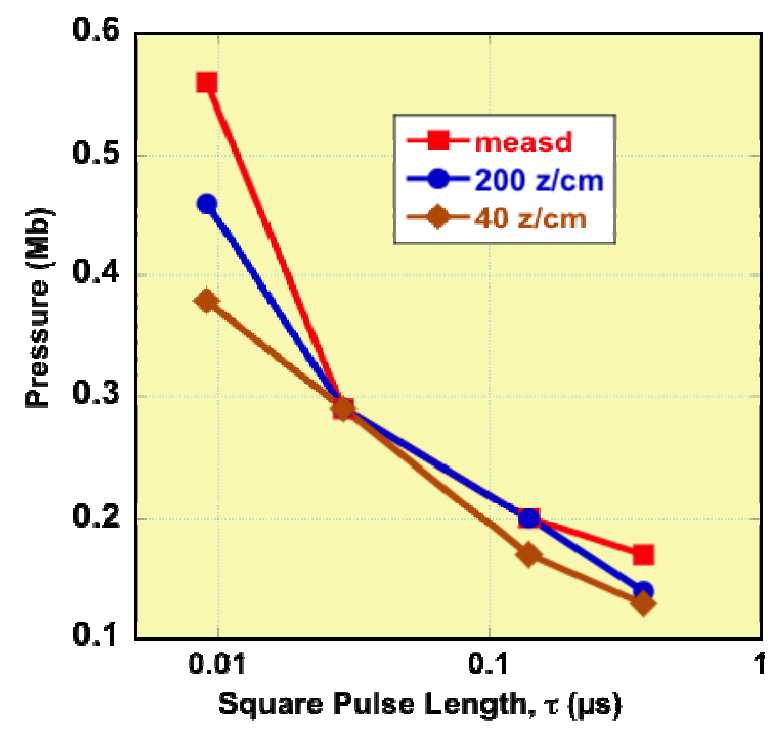

Table 6a. 50-50 Pressure-pulse times for initiation of LX-17. Detonation occurs above the lines and failure below. The thin pulse region lies to the upper left; the bullet region lies to the lower right.

\section{Crossing Air Gaps}

Most of the ambient LX-17 experiments were 1-inch diameter, made up of 1" x 1" pellets, always with a Comp B pellet at the start [Souers2]. This was followed by a donor section of LX-17, which varied from 1 to 6 pellets $(25.4 \mathrm{~mm}$ to $152.4 \mathrm{~mm})$. Then came a variable -width air gap, followed by 4 to 6 pellets of LX-17. This long acceptor section was necessary to ensure that the detonated restarted. Pins were placed along the acceptor section to measure the detonation velocity. All these shots were bare, with the pellets lying on a rack. A single 2 inch copper-confined shot was also done. All modeling was done with relaxation in the air gap from zero time on.

The GO-NO GO, 1-inch results are shown in the top section of Table 7a. The critical gap lengths (ie. between the largest GO and the smallest NO GO) increase slightly as the donor length increases. The calculated critical gaps are all smaller. Using no relaxation in the gaps appears to lengthen the critical gap, but the model tangles badly. Below in Table $7 a$ is the time delay data. This was obtained by comparing times from the start of the gap between nogap and gapped ratesticks. Again, the entire length of the acceptor was needed to make the delay level off. 
Table 7a. Summary of air gap crossing data for the bare inch size.

\begin{tabular}{|c|c|c|c|c|}
\hline & & \multicolumn{3}{|c|}{ Listed Donor Length (mm) } \\
\hline \multicolumn{2}{|l|}{ zones/cm } & 25.4 & 50.8 & 152.4 \\
\hline \multicolumn{2}{|l|}{ Measured } & 2.3 & 3.5 & 3.8 \\
\hline 40 & & 1.9 & 2.1 & 2.6 \\
\hline \multirow[t]{3}{*}{200} & & 1.9 & 2.1 & \\
\hline & & \multicolumn{2}{|c|}{ Delay $(\mu \mathrm{s})$ for Listed Donor } & \\
\hline & & & Length (mm) & \\
\hline \multirow{11}{*}{\begin{tabular}{|c|} 
zones $/ \mathrm{cm}$ \\
40
\end{tabular}} & gap $(\mathrm{mm})$ & 25.4 & 50.8 & 152.4 \\
\hline & 0.50 & & & \\
\hline & 0.75 & 0.070 & & \\
\hline & 1.00 & 0.095 & 0.24 & \\
\hline & 1.25 & 0.23 & & \\
\hline & 1.50 & 0.66 & 0.42 & \\
\hline & 1.75 & 0.99 & & \\
\hline & 2.00 & & 0.80 & 0.65 \\
\hline & 2.25 & & & 0.90 \\
\hline & 2.50 & & & 0.90 \\
\hline & 2.75 & & & \\
\hline \multirow{11}{*}{\begin{tabular}{|c|} 
zones/cm \\
200 \\
\end{tabular}} & gap $(\mathrm{mm})$ & 25 & 50 & 150 \\
\hline & 0.50 & 0.15 & & \\
\hline & 0.75 & & & \\
\hline & 1.00 & 0.28 & & \\
\hline & 1.25 & & & \\
\hline & 1.50 & & & \\
\hline & 1.75 & 1.06 & & \\
\hline & 2.00 & & 0.75 & \\
\hline & 2.25 & & & \\
\hline & 2.50 & & & \\
\hline & 2.75 & & & \\
\hline
\end{tabular}

The comparison of measured and calculated delay times are shown in Figure 7a. The delay time is slightly longer the longer is the donor, and this difference is seen in the calculations. The measurements are scattered enough that we just plot everything together.

The time delays, $t_{d}$ (in $\mu s$ ), are approximately

$$
\begin{aligned}
& \mathrm{t}_{\mathrm{d}}(1-\text { inch bare }) \approx 0.26 \mathrm{~g} \\
& \mathrm{t}_{\mathrm{d}}(2-\text { inch confined }) \approx 0.093 \mathrm{~g}
\end{aligned}
$$




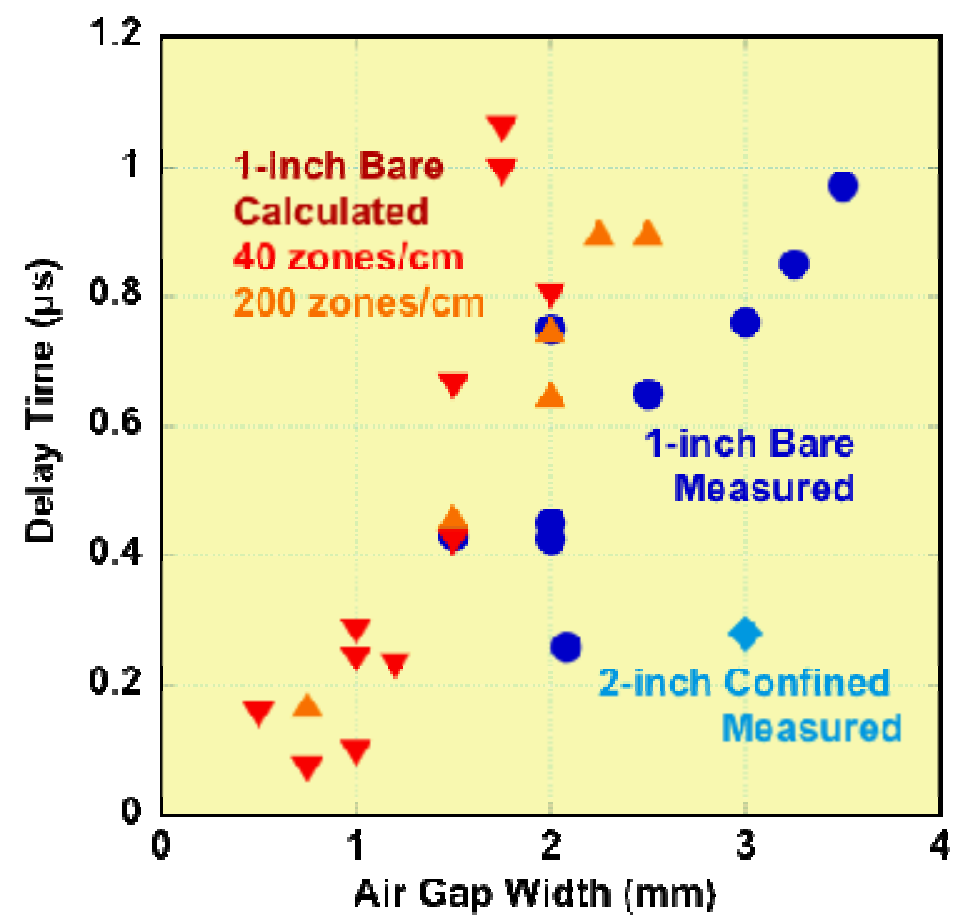

Figure 7a. Delay times for the bare1-inch ratesticks, both calculated and measured. A single confined 2-inch value is also shown.

where $\mathrm{g}$ is the gap width in $\mathrm{mm}$. Only one point makes up the second equation. These equations can be used to estimate delays in small gaps.

\section{Jack Rabbit \#3 Corner Turn}

Jack Rabbit \#3 is the smallest of a series of five corner-turning shots by Mark Hart of LLNL [Hart1 to Hart7]. We use \#3 partly because it is the smallest and also because it showed no unusual time delays see, for example, the $2 \mu$ s delay in \#4). The schematic is shown in Figure 8a. The detonation blows to the right and up, going around the steel plate with the formation of a dead zone at the edge. Some of it runs around to the back but a weak shock is also transmitted to the left through the steel. Eventually, the left hand aluminum moves out, with PDV's watching all along the surface. 


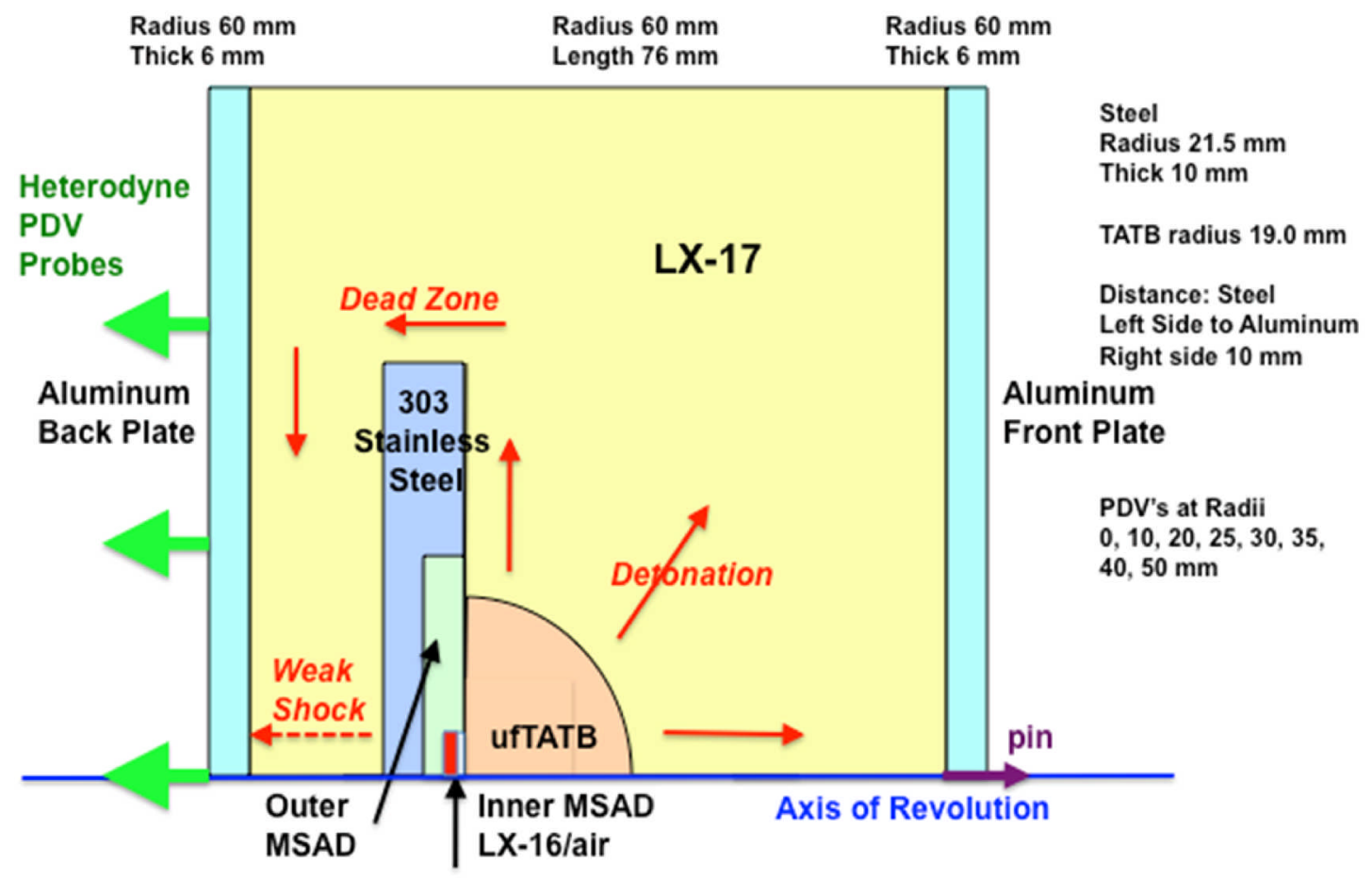

Table 8a. Schematic of Jack Rabbit3.

The detonation front at the time of corner-turning at 40 zones/cm is shown with pseudocolored density In Figure 8b. The dead zone at the top of the steel is evident. The detonation front in the other directions looks too thick and has a hitch in it, so that Tarantula is having trouble with the divergence.

Figure $8 c$ shows the results of the aluminum plate velocities at 40 zones $/ \mathrm{cm}$. The calculated result (bold) is higher than the measured (light), and this has occurred in every Tarantula/JWL++ model. If we turn on the desensitization model to

$$
\text { desenz rate } \approx 5, \quad 0<(P+Q)<0.05 \mathrm{Mb} \text {, }
$$

we make some LX-17 inert. Eq. 1 is completely empirical, but it does diminish the effect of the weak shock moving back through the steel directly to the aluminum plate. Using Eq. 1 will lower all the calculated velocities of Figure $8 \mathrm{c}$ down into the region of the data. Whether this is physically justified is unknown. 


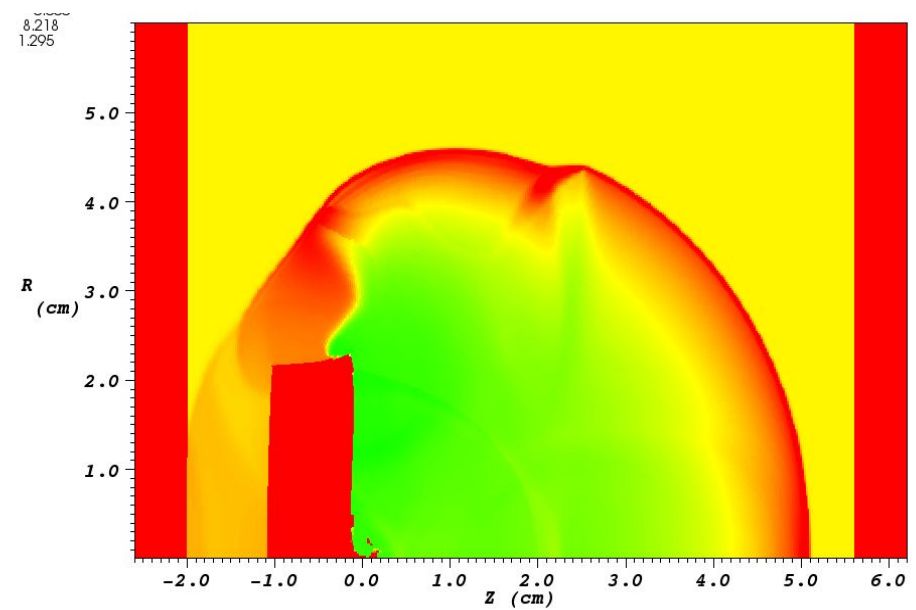

Figure 8b. Pseudo-color density figure for Jackrabbit 3 with the dead zone at the left. The detonator is at $(0,0)$.

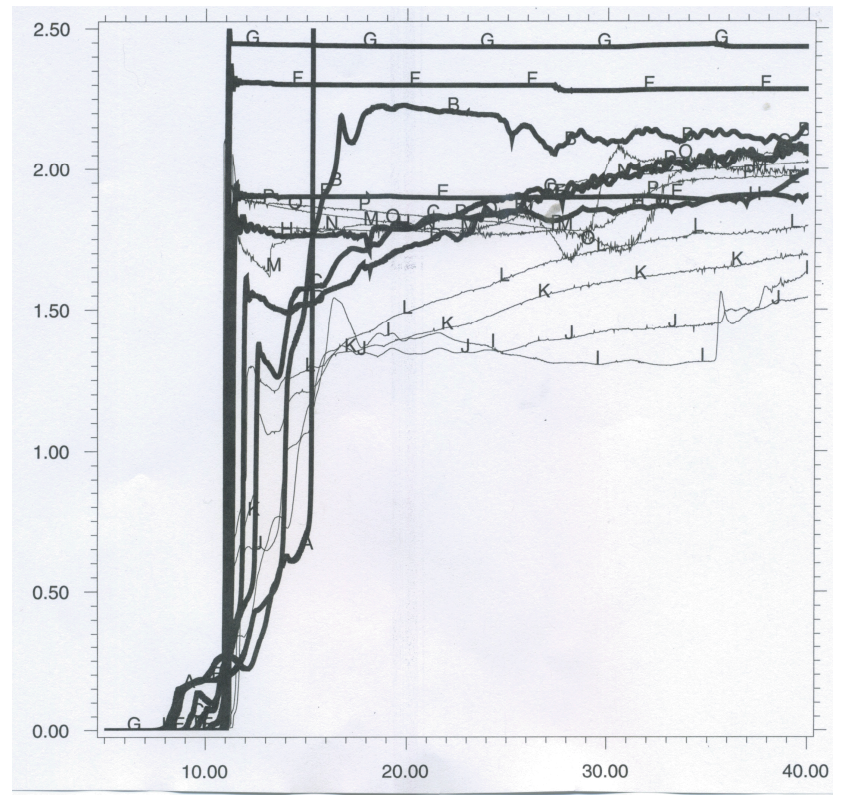

Figure 8c. Velocity of the aluminum plate. The code is bold and the data is light.

Desensitization can be turned on to lower the calculated velocities on top of the measured ones. 


\section{Difficulties with Hemispheres in Square Zones}

We next added the hemisphere geometries with square zoning. The experiment was Chadd May's ambient LX-10 booster driving an LX-17 snowball with an MSAD for initiation [May]. The LX-10 booster radius was changed so that full breakout occurred with a $6.5 \mathrm{~mm}$ radius $L X-10$ and complete failure occurred with a $4.0 \mathrm{~mm}$ radius with $5.0 \mathrm{~mm}$ being an intermediate "eat-a-hole" case. This data, done only at room temperature, constitutes a serious challenge for any new all-purpose model. Figure 9a shows the measured breakout for different radius boosters.

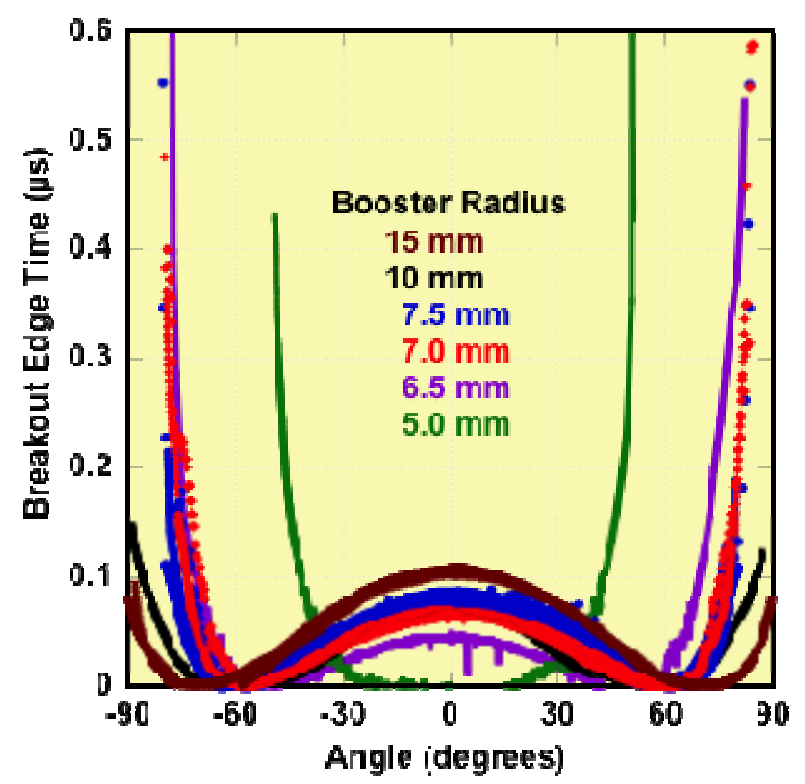

Figure 9a. Chadd May's LX-17 snowball breakout with different-radius LX-10 boosters. The $4.0 \mathrm{~mm}$-radius booster fails to light the LX-17. The $5.0 \mathrm{~mm}$ booster "eats-a-hole" in the LX-17.

The model results are shown in Table 9a. The $4.0 \mathrm{~mm}$ case always fails as it is supposed to, but the $6.5 \mathrm{~mm}$ case is very weak with mostly dead zones and a front only over a small number of degrees. A sample is shown in Figure $9 \mathrm{~b}$. At 40 zones $/ \mathrm{cm}$, the $6.5 \mathrm{~mm}$ case dies if carried out beyond the $21.5 \mathrm{~mm}$ original radius. Oddly, the booster times at the $\mathrm{k}$ edit at $50^{\circ}$ are not bad. It is supposed to be $3.1 \mu$ s and they get closer as the zoning increases.

Table 9a. Model results for the LX-10/LX-17 snowball in square zoning. OK 45-50 means we get a front only between 40 and 50 degrees and dead zones otherwise.

\begin{tabular}{|c|c|c|c|c|c|}
\hline $\begin{array}{c}\text { zones/ } \\
\mathrm{cm}\end{array}$ & $\begin{array}{c}6.5 \mathrm{~mm} \\
\mathrm{k} \text { time }(\mu \mathrm{s})\end{array}$ & $\begin{array}{c}6.5 \mathrm{~mm} \\
\text { result }\end{array}$ & $\begin{array}{c}4.0 \mathrm{~mm} \\
\text { result }\end{array}$ & $\begin{array}{c}12.5 \mathrm{~mm} \\
\text { result }\end{array}$ & $\begin{array}{c}15.0 \mathrm{~mm} \\
\text { result }\end{array}$ \\
\hline 40 & 2.63 & ok $40-50$ & big FAlL & ok $0-70$ & full curve \\
\hline 200 & 2.94 & ok $40-50$ & FAlL & full curve & full curve \\
\hline 360 & 3.18 & FAlL & slow FAlL & ok $10-70$ & ok $0-80$ \\
\hline
\end{tabular}


So it turns out, with square zones, that Tarantula2011 is too weak to adequately drive divergent detonation with the cylinder parameters. Worse yet, the difference in behavior between 6.5 and $4.0 \mathrm{~mm}$ is too small, so that the failure mechanism is not working. It is like simple JWL++ in the cylinder where the quadratic rate cannot shut down between $6.5 \mathrm{~mm}$ radius and $2 \mathrm{~mm}$. This situation is not improved with increased zoning, and possibly, it becomes worse, and no amount of fiddling with the parameters has improved it. JWL++ or Ignition \& Growth with a simple quadratic rate both have no trouble driving hemispheres, but neither can they do cylinder dead zones or failure at the same time. For this, the Reactive Flow needs to be weakened and this affects divergent flow.

Also shown in Table 9a are the runs with larger boosters, and it takes a $15 \mathrm{~mm}$-radius booster to ensure that we get a good-looking detonation front. This is a graphic measure of how weak the reactive flow is, and it means that no small adjustment will fix the problem.

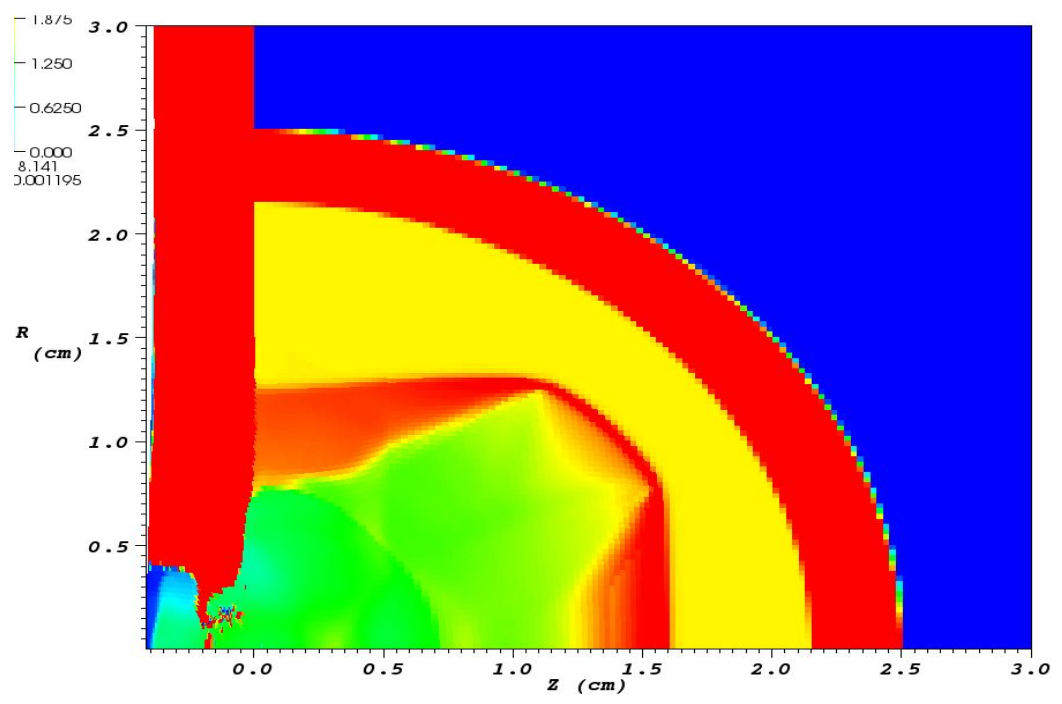

Figure $9 \mathrm{~b}$. The $6.5 \mathrm{~mm}$-radius booster problem at 40 zones/cm: too much dead zone. The red on the left is steel and the curved red part is lithium fluoride.

\section{The Hemisphere Problem with Butterfly Zoning}

We experimented with different zoning schemes while keeping the cylinder-derived calibration. Simply elongating zones in either direction from the square format does not work, and the detonation fails. We then tried the butterfly mesh shown in Figure 10a. The MSAD is at the lower left and is explicity modeled. It hits the $L X-10$ booster in a square containing 
square zoning. This spreads out into a butterfly mesh, which has a dividing line running at $45^{\circ}$. Finally it blends into radial zoning. It was found that the central square had to be kept small and the radial zones had to be elongated in the radial direction in order to get a good answer. This allowed the detonation to propagate somewhat better in the upward dir ection.

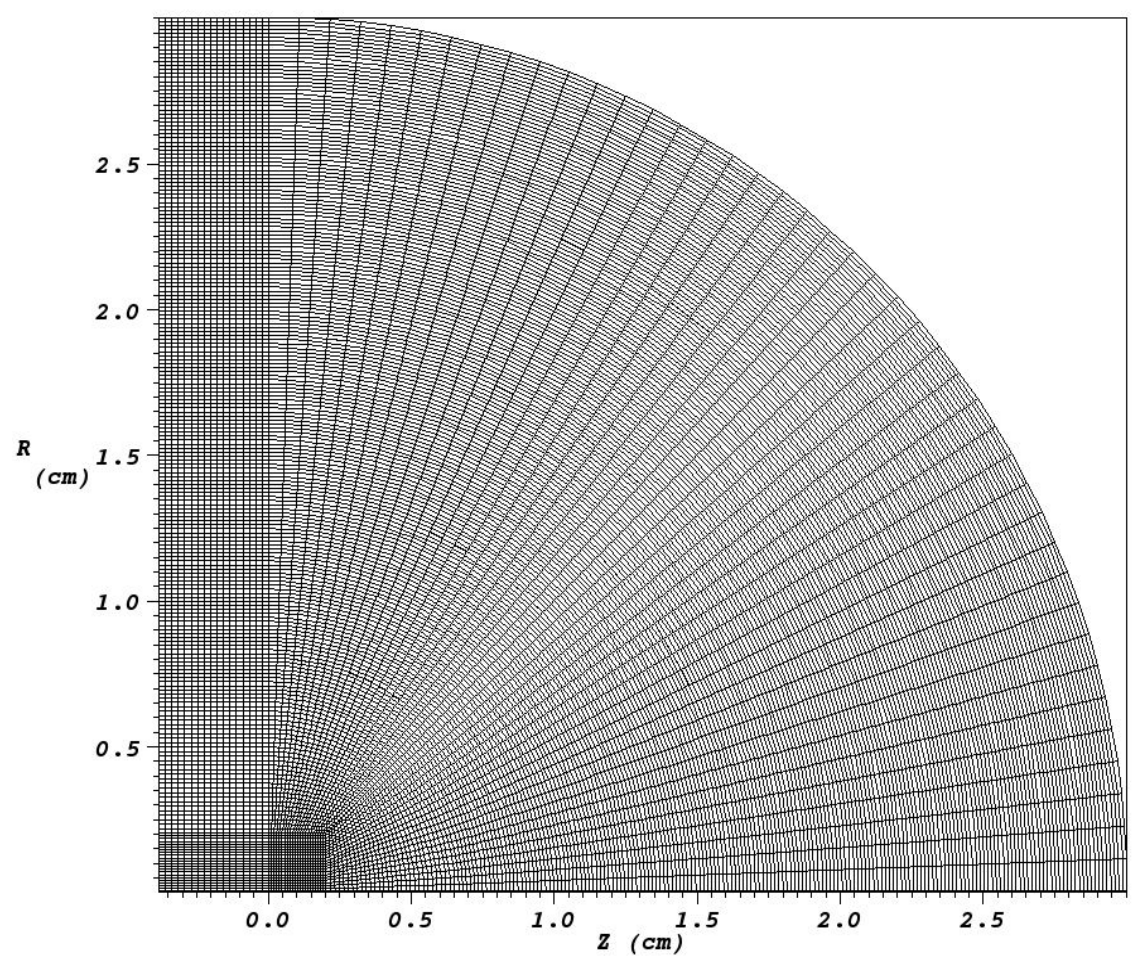

Figure 10a. Square zones out to $0.2 \mathrm{~cm}$; butterfly mesh to $0.65 \mathrm{~cm}$, radial zoning outside. The elongated radial zones are needed to get a good answer. The MSAD is modeled explicitly at the lower left.

A final design is shown in Figure 10b. We here go from the central square to the butterfly to the radial, back to the butterfly and finally to square zoning again. This allows insertion of the detonator/booster region while maintaining square zones at large distances in a very big main charge. 


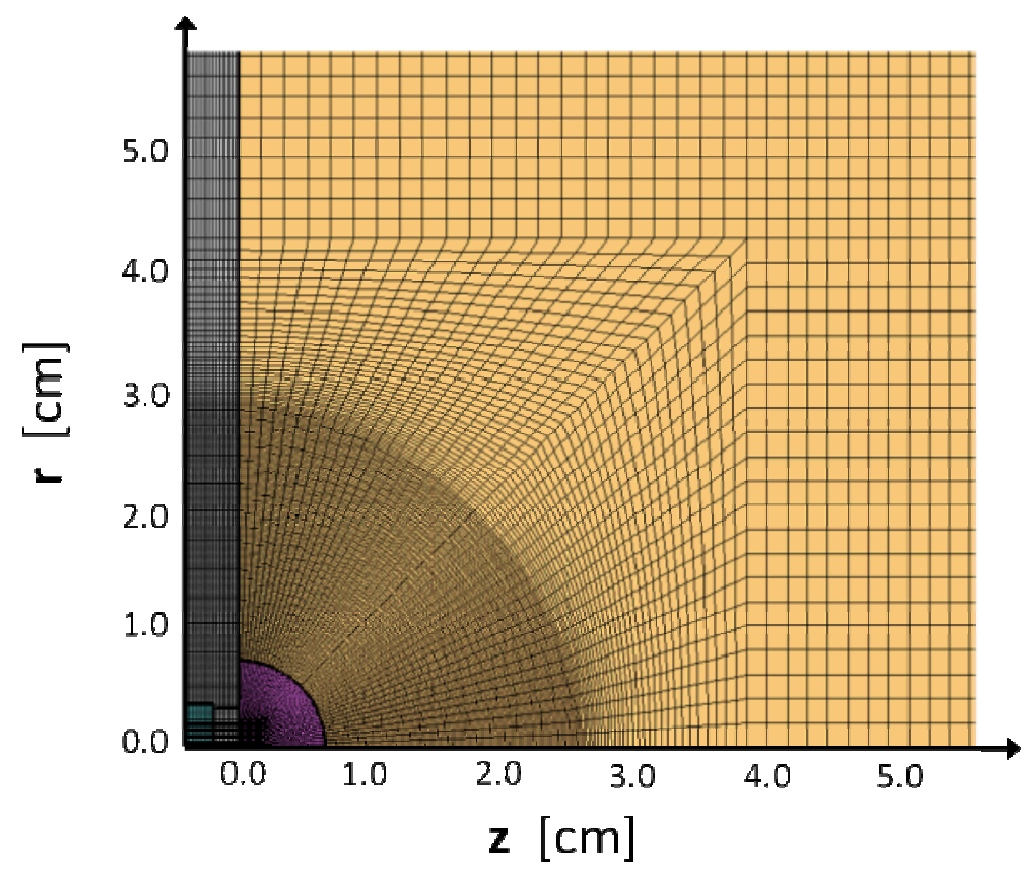

Figure 10b. Square inner box with butterfly conversion to radial followed by a butterfly conversion back to square zones. The MSAD is modeled explicitly at the lower left.

The results are summarized in Table 10a. At the top are the results of the square zoning studies from the last section. At the bottom are the butterfly meshes. The first two are like Figure 10a with 40 and 200 zones/cm cylindrical calibrations. The third one is Figure $10 \mathrm{~b}$ with 40 zones/cm cylindrical calibration. The third one has 800 zones $/ \mathrm{cm}$ in a $0.20 \mathrm{~cm}$ square box, transitions to $60 \times 80$ zones $/ \mathrm{cm}$ butterflies, and ends with 40 zones $/ \mathrm{cm}$. The calibration belongs to the final zoning in this case. We see that the reactive flow is still weak.

Table 10a. LX-10/LX-17 snowball results with square zoning (top) and Butterfly meshes (bottom).

\begin{tabular}{|c|c|c|}
\hline zones/ & $6.5 \mathrm{~mm}$ & $4.0 \mathrm{~mm}$ \\
\hline $\mathrm{cm}$ & result & result \\
\hline 40 & ok $40-50$ & big FAlL \\
\hline 200 & ok $40-50$ & FAlL \\
\hline 360 & FAlL & slow FAIL \\
\hline $10 \mathrm{a}-40$ & ok $0-60$ & FAlL \\
\hline 10 ba-200 & ok $0-60$ & FAlL \\
\hline $10 \mathrm{~b}-40$ & ok $0-70$ & FAlL \\
\hline
\end{tabular}

The listed butterfly meshes fail every time at $4 \mathrm{~mm}$ as they are supposed to. At $6.5 \mathrm{~mm}$, the front is better than it was in square zoning but still possesses large dead zones and so is not the desired answer. The front from the third butterfly mesh is shown in Figure 10c. It is 
not bad but a dead zone remnant lingers on. The first runs with Tarantula2012 suggest there is a better way to attack this problem in the more desirable square meshes.

Another problem with this approach is that other geometries, like the double cylinder, also have a component of divergence. Figure $10 \mathrm{~d}$ shows the extension of the butterfly mesh to corner-turning. The dead zone is still there but the detonation front is ragged.

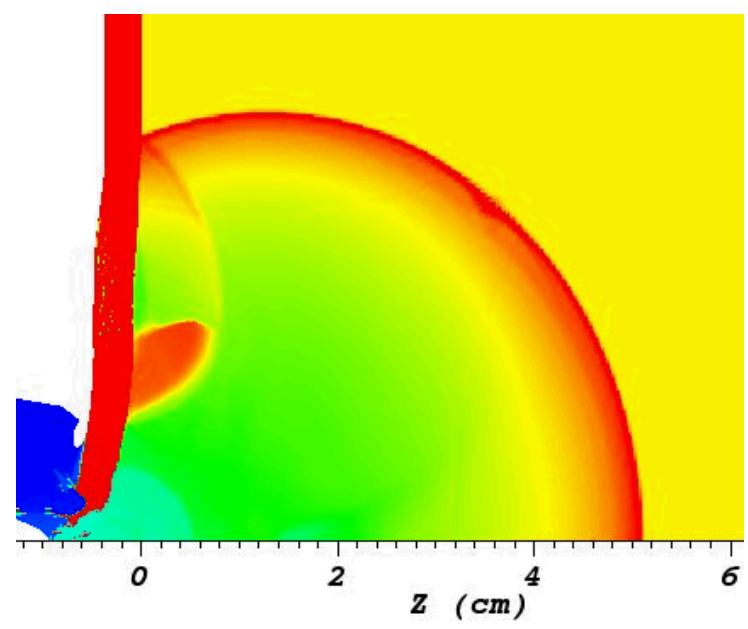

Figure 10c. Detonation front from the third butterfy mesh. A dead zone lingers on at the left.
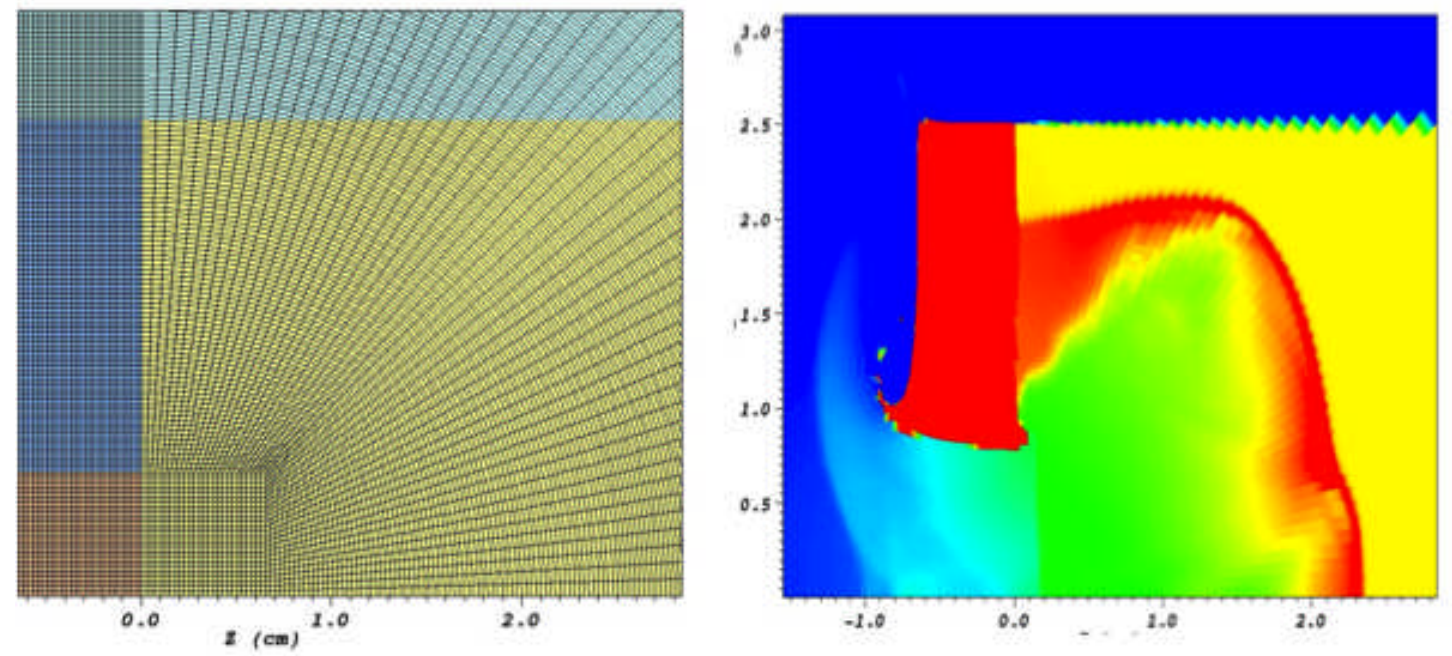

Figure 10d. Butterfly mesh for the double cylinder with resulting dead zone and a perturbed detonation front. 


\section{References}

[Campbell] A. W. Campbell, "Diameter Effect and Failure Diameter of a TATB-Based Explosive," Propellants, Explosives, Pyrotechnics, 9, 183-187 (1984).

[Dallman] D. C. Dallman and Jerry Wackerle, "Temperature-Dependent Shock Inititation of TATB-Based Explosives," Proceedings Tenth Symposium (International) on Detonation, Boston, MA, July 12-16, 1993, pp. 130-138.

[Gustavsen] R. L. Gustavsen, S. A. Sheffield, R. R. Alcon, J. W. Forbes, C. M. Tarver and F. Garcia, "Embedded Electromagnetic Gauge Measurements and Modeling of Shock Initiation in the TATB Based Explosives LX-17 and PBX 9502," Shock Compression of Condensed Matter- 2001, M. D. Furnish, N. N. Thadhani and Y. Horie, American Institute of Physics (2002, pp. 1019-1022.

[Hart1] Mark M. Hart, "Jack Rabbit Investigation of TATB IHE Detonation Chemical Kinetics," Proceeding Fourteenth International Detonation Symposium, Coeur d'Alene, ID, April 1116, 2010, pp. 282-291.

[Hart2] M. M. Hart and D. W. McDaniel, Jack Rabbit Pretest Shadowplate Drawings for TATB IHE Model Development, Volume 9, LLNL report LLNL-TR-1415545 (2009).

[Hart3] M. M. Hart, O. T. Strand and S. T. Bossen, "Jack Rabbit Pretest 2021E PT3 Photonic Doppler Velocimetry Data, Volume 3, LLNL report LLNL-TR-404999 (2008).

[Hart4] M. M. Hart, O. T. Strand and S. T. Bossen, "Jack Rabbit Pretest 2021E PT4 Photonic Doppler Velocimetry Data, Volume 3, LLNL report LLNL-TR-405000 (2008).

[Hart5] M. M. Hart, O. T. Strand and S. T. Bossen, "Jack Rabbit Pretest 2021E PT5 Photonic Doppler Velocimetry Data, Volume 5, LLNL report LLNL-TR-405002 (2008).

[Hart6] M. M. Hart, O. T. Strand and S. T. Bossen, "Jack Rabbit Pretest 2021E PT6 Photonic Doppler Velocimetry Data, Volume 6, LLNL report LLNL-TR-405004 (2008).

[Hart7] M. M. Hart, O. T. Strand and S. T. Bossen, "Jack Rabbit Pretest 2021E PT7 Photonic Doppler Velocimetry Data, Volume 7, LLNL report LLNL-TR-405006 (2008).

[Hill1] L. G. Hill, J. D. Bdzil and T. D. Aslam, "Front Curvature Rate Stick Measurements and Calibration of the Detonation Shock Dynamics Model for PBX 9502 over a Wide Temperature Range," Eleventh International Detonation Symposium, Snowmass Village, CO, August 31-September 4, 1999, pp. 1029-1037.

[Hill2] Larry Hill and Maria Rightley, Los Alamos National Laboratory, Los Alamos, NM, private communication, 2001.

[Hill3] L. G. Hill, J. D. Bdzil, W. C. Davis and R. R. Critchfield, "PBX 9502 Front Curvature Rate Stick Data: Repeatability and the Effects of Temperature and Material Variation," Thirteenth International Detonation Symposium, Norfolk, VA, July 23-28, 2006, pp. 33134. 
[Honodel1] C. A. Honodel, J. R. Humphrey, R. C. Weingart, R. S. Lee and P. Kramer, "Shock Initiation of TATB Formulations," Proceedings Seventh Symposium (International) on Detonation, Annapolis, MD, June 16-19, 1981, pp. 425-434.

[Honodel2] Raw data of R. C. Weingart found and reanalyzed by P. C. Souers, 2004. The data is previously reported in [Honodel1].

[Hutchinson] C. D. Hutchinson, G. C. W. Foan, H. R. Lawn and A. G. Jones, "Initiation and Detonation Properties of the Insensitive High Explosive TATB/Kel-F 800 95/5", Proceedings Ninth Symposium (International) on Detonation, Portland, OR, August 28September 1, 1989, pp. 123-132.

[Jackson] R. K. Jackson, L. G. Green, R. H. Barlett, W. W. Hofer, P. E. Kramer, R. S. Lee, E. J. Nidick, Jr., L. L. Shaw and R. C. Weingart, Proceedings Sixth Symposium (International) on Detonation, Coronado, CA, August 24-27, 1976, pp. 755-765.

[May] Chadd May, LLNL, private communication, 2009.

[Phillips] Dan Phillips and Chadd May, LLNL, private communication to P. C. Souers, 2010.

[Souers1] P. C. Souers, LLNL Cylinder Test data library.

[Souers2] P. Clark Souers, Lisa Lauderbach, Raul Garza, Peter Vitello and David E. Hare, "LX-17 and ufTATB Data for Corner-Turning, Failure and Detonation," $14^{\text {th }}$ International Detonation Symposium, Coeur d"Alene Resort, Idaho, April 11-16, 2010, 1237-1245. 